OPEN ACCESS

Edited by:

Guochun Wang,

China-Japan Friendship Hospital,

China

Reviewed by:

Xavier Bossuyt,

KU Leuven, Belgium

Christopher Richardson,

University of Rochester, United States

*Correspondence:

Ben Mulhearn

bm765@bath.ac.uk

Specialty section: This article was submitted to

Autoimmune and

Autoinflammatory Disorders,

a section of the journal

Frontiers in Immunology

Received: 28 October 2021

Accepted: 12 January 2022

Published: 28 January 2022

Citation:

Mulhearn B, Li D, McMorrow F,

Lu H, McHugh NJ and Tansley SL

(2022) A Commercial Anti-TIF1 $\gamma E L I S A$

Is Superior to Line and Dot Blot

and Should Be Considered as

Part of Routine Myositis-Specific

Antibody Testing.

Front. Immunol. 13:804037.

doi: 10.3389/fimmu.2022.804037

\section{A Commercial Anti-TIF1 $\gamma$ ELISA Is Superior to Line and Dot Blot and Should Be Considered as Part of Routine Myositis-Specific Antibody Testing}

\author{
Ben Mulhearn ${ }^{1,2 *}$, Danyang $\mathrm{Li}^{1}$, Fionnuala McMorrow ${ }^{1}$, Hui Lu ${ }^{1}$, Neil J. McHugh ${ }^{1}$ \\ and Sarah L. Tansley ${ }^{1,2}$ \\ ${ }^{1}$ Department and Pharmacy and Pharmacology, University of Bath, Bath, United Kingdom, ${ }^{2}$ Royal National Hospital for \\ Rheumatic Diseases, Royal United Hospitals, Bath, United Kingdom
}

Objectives: Anti-TIF1 $\gamma$ is an important autoantibody in the diagnosis of cancerassociated dermatomyositis and the most common autoantibody in juvenile onset dermatomyositis. Its reliable detection is important to instigate further investigations into underlying malignancy in adults. We previously showed that commercial assays using line and dot blots do not reliably detect anti-TIF1 $\gamma$. We aimed to test a new commercial ELISA and compare with previously obtained protein immunoprecipitation.

Methods: Radio-labelled immunoprecipitation had previously been used to determine the autoantibody status of patients with immune-mediated inflammatory myopathies and several healthy controls. ELISA was undertaken on healthy control and anti-TIF1 $\gamma$ sera and compared to previous immunoprecipitation data.

Results: A total of 110 serum samples were analysed: 42 myositis patients with anti- TIF1 $\gamma$ and 68 autoantibody negative healthy control sera. Anti-TIF1 $\gamma$ was detected by ELISA in 41 out of 42 of the anti-TIF1 $\gamma$-positive samples by immunoprecipitation, and in none of the healthy controls, giving a sensitivity of $97.6 \%$ and specificity of $100 \%$. The false negative rate was $2 \%$.

Conclusion: ELISA is an affordable and time-efficient method which is accurate in detecting anti-TIF1 $\gamma$.

Keywords: TIF1 $\gamma$, cancer, autoantibodies, myositis, ELISA - enzyme-linked immunosorbent assay, myositis diagnosis, dermatomysitis

\section{HIGHLIGHTS}

1. Anti-TIF1 $\gamma$ is a key autoantibody in the diagnosis of cancer-associated dermatomyositis and juvenile dermatomyositis

2. ELISA is a quick and easy method in accurately detecting anti-TIF1 $\gamma$ autoantibodies

3. Diagnosis of IIMs should include ANA immunofluorescence, line or dot blot, and anti-TIF1 $\gamma$ ELISA 


\section{INTRODUCTION}

The ability to detect myositis -specific and -associated antibodies (MSAs and MAAs), which can be found in the sera of $60-70 \%$ patients with immune-mediated inflammatory myopathies (IIMs) (1), has greatly improved the diagnosis and phenotyping of these rare diseases. Not only do they aid diagnosis, but they also guide further investigation and management (2). For instance, it is well-known that IIMs, and dermatomyositis (DM) in particular, are strongly linked with cancer, with estimates varying between 7 and 32\% (3).

Anti-transcription intermediary factor $1 \gamma(\mathrm{TIF} 1 \gamma)$ autoantibodies are found in both juvenile dermatomyositis (JDM) and adult IIMs. They are present in 7\% of European adults with DM and $20-30 \%$ of children affected by JDM (2). Strikingly, $38-84 \%$ of patients adult DM patients $\geq 39$ years of age who are TIF1 $\gamma$-positive in both European and Japanese cohorts develop cancer in the 3 years before and after DM diagnosis (4-6). Anti-TIF1 $\gamma$ detection in patients with a new diagnosis of $\mathrm{DM} \geq 39$ years of age may therefore prompt a thorough investigation for the detection of cancer and reduce cancer mortality rates, making the accurate detection of anti-TIF1 $\gamma$ a research priority.

Currently the reference standard in the detection of MSAAs is immunoprecipitation (IP) due to its ability to detect well-described and novel autoantibodies. However, this technique is impractical for use in clinical practice owing to its expense and the length of time it takes to reach a result which usually takes a minimum of $2-3$ weeks. For this reason, several commercially available immunoassays have become available which are low cost, easy to use, and are reported to detect an array of MSAAs. However, these immunoassays are subject to both false positives and false negatives. A number of them have recently been tested by our group and others $(7,8)$. In particular, antiTIF1 $\gamma$ was found to be particularly problematic with false negatives found in $40 \%$ samples analysed by line blot and $76 \%$ by dot blot (7). Espinosa-Ortega et al. (8) also found low concordance between antiTIF1 $\gamma$ detected by line/dot blot and immunoprecipitation, with a Cohen's kappa of 0.56 . This is likely because anti-TIF1 $\gamma$ frequently target a conformational epitope, meaning the tertiary antigen structure is required to remain intact to be recognised by the autoantibody (9). Whereas line and dot blot immunoassays utilise denatured antigen, enzyme-linked immunosorbent assays (ELISAs) maintain the tertiary structure of the protein. Fujimoto et al. (10) recently tested a newly-developed ELISA in a Japanese cohort of patients with a spectrum of IIMs, and found this approach to be highly effective with $100 \%$ sensitivity and specificity which was a result comparable to immunoprecipitation.

In this study, we aimed to test the same commercial ELISA kit (Medical \& Biological Laboratories Co. Ltd., Nagoya, Aichi, Japan) for the detection of TIF1 $\gamma$ autoantibodies in a European cohort of adult IIM patients and compared results with samples previously analysed using immunoprecipitation.

\section{METHODS}

\section{Sample Selection}

Myositis serum samples used in this study were chosen as previously described (7) from a biobank of more than 3000 samples collected for research or diagnostic purposes $(2,11)$. All serum samples had previously been analysed by immunoprecipitation locally and contain at least one MSAA. Twenty-five anti-TIF1 $\gamma$ samples had also been previously analysed by line and dot blot (7). Briefly, sera were stored at $-20^{\circ} \mathrm{C}$ prior to analysis in a facility at the University of Bath. The study had ethical approval through the host Institute (University of Bath EIRA reference number 17-01211). All samples from research cohorts had existing ethics in place.

\section{ELISA}

ELISA was performed on $5 \mu \mathrm{L}$ of diluted serum sample as per the manufacturer's instructions (Medical \& Biological Laboratories Co. Ltd., Nagoya, Aichi, Japan). All samples were run in duplicate. Briefly, samples were thawed and diluted to a 1:101 concentration and incubated on a microwell plate for 30 minutes. Wells were then incubated with a horseradish peroxidase-conjugated goat antihuman IgG antibody conjugate for 30 minutes followed by a TMB/ peroxide substrate for 15 minutes. The reaction was terminated by $0.25 \mathrm{~mol} / \mathrm{L}$ sulfuric acid. All incubations took place at room temperature with 4 wash cycles between steps. The absorbance of each well was read on a FLUOstar Omega microplate reader (BMG Labtech Ltd., Aylesbury, Buckinghamshire, Great Britain) at $450 \mathrm{~nm}$ wavelength. Positive and negative cut off values were calculated according to previous work described by Fujimoto et al. (10) and expressed in arbitrary units (au).

\section{Immunoprecipitation}

Radio-immunoprecipitation had been previously undertaken as described by Tansley et al. (7). Briefly, sera were mixed with protein-A-Sepharose beads and a 35(S)methionine radiolabelled K562 cell extract, followed by fractionation by SDS-PAGE and analysis by autoradiography. A characteristic doublet band at $155 / 140$ was read as being positive for TIF1 $\gamma(12)$.

\section{Data Analysis}

Statistical analysis was undertaken using Prism 9 version 9.2.0 for macOS (GraphPad Software, LLC., San Diego, CA, USA). Confidence intervals (CI) are expressed at 95\%.

\section{RESULTS}

A total of 110 serum samples were analysed, of which 42 were known to have anti-TIF1 $\gamma$ and 68 were healthy control sera. Immunoprecipitation data was held for all samples. Diagnoses included DM $(n=27)$, clinically amyopathic DM $(n=4), \operatorname{JDM}(n=5)$, polymyositis $(n=4)$, and overlap syndrome $(n=2)$. All HC samples tested were autoantibody negative by immunoprecipitation.

\section{Commercial TIF1 $\gamma$ ELISA Performed as Well as Immunoprecipitation}

Forty-one patient samples with anti-TIF1 $\gamma$ tested positive by ELISA as defined by a cut-off point of $32 \mathrm{au}$. None of the HC samples tested positive using this cut-off point. The remaining anti-TIF1 $\gamma$ positive sample was just under the cut-off for positivity (30.2 au). This gives an area under the ROC curve 
(AUC) of 0.988 (CI $0.961-1.000, \mathrm{P}<0.0001$ ) which is equivalent to sensitivity of $97.6 \%$ (CI $87.7 \%-99.9 \%$ ) and a specificity of $100 \%$ (CI 94.65\% - 100\%). In this case, Cohen's Kappa would give a value of 1 .

Quantitative results for the ELISA values are shown in Figure 1. Briefly, the median ELISA assay result for $\mathrm{HC}$ samples was 5.99 au. (median CI 4.74 - 7.87) and for the TIF1 $\gamma$ samples was 128.5 au. (median CI 110.4 - 135.4).

Graph showing the relative ELISA titres for healthy control and TIF1 $\gamma$ samples expressed in arbitrary units for each individual serum sample (circles). Dashed line represents the positive cut-off point as previous described (10). All 68 healthy control (HC) samples were underneath the cut-off and all but one of the 42 TIF1 $\gamma$ samples were above the cut-off. The TIF1 $\gamma$ sample below the cut-off had a weak band in the $140 / 155 \mathrm{kDa}$ region.

\section{Low Anti-TIF1 $\gamma$ ELISA Titres Are Associated With False Negative Line Blot Results}

Given that our group previously tested 25 anti-TIF $1 \gamma$ samples by line blot, we were able to compare ELISA titres in this study with this data to try and understand which samples might test negative by line blot. The results are shown in Figure 2. All anti-TIF1 $\gamma$ positive samples by ELISA with low titres (between 30 - 100 au.) tested negative by line blot. However, 3 out of the 9 samples testing negative by line blot had high anti- TIF1 $\gamma$ titres (> $100 \mathrm{au}$.). The difference in ELISA titres between those testing negative and positive by line blot was statistically significant $(\mathrm{P}=$ 0.0041, two-tailed Mann-Whitney test), suggesting that lower anti-TIF1 $\gamma$ antibody titres lead to false negative line blot results. Similarly, dot blot samples returned only 7/24 (29\%) true

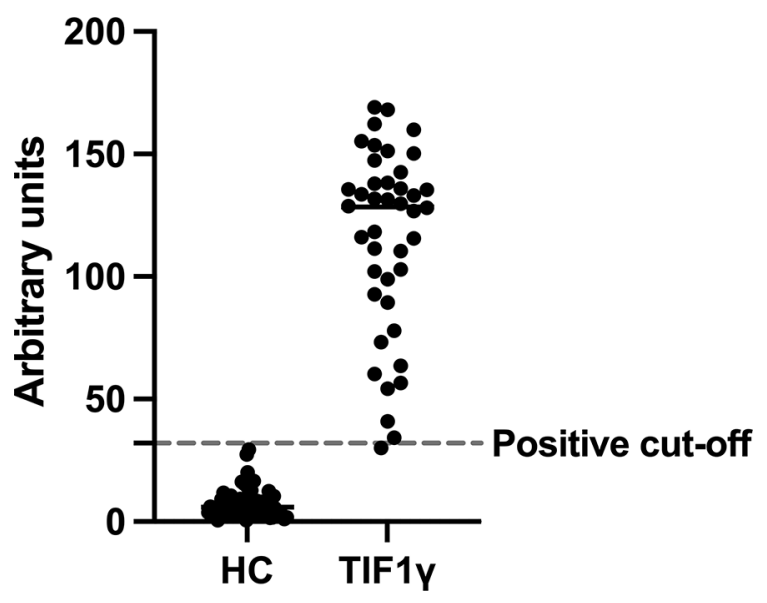

FIGURE 1 | TIF1 $\gamma$ ELISA values for 68 healthy controls and 42 TIF1 $\gamma$ serum samples.

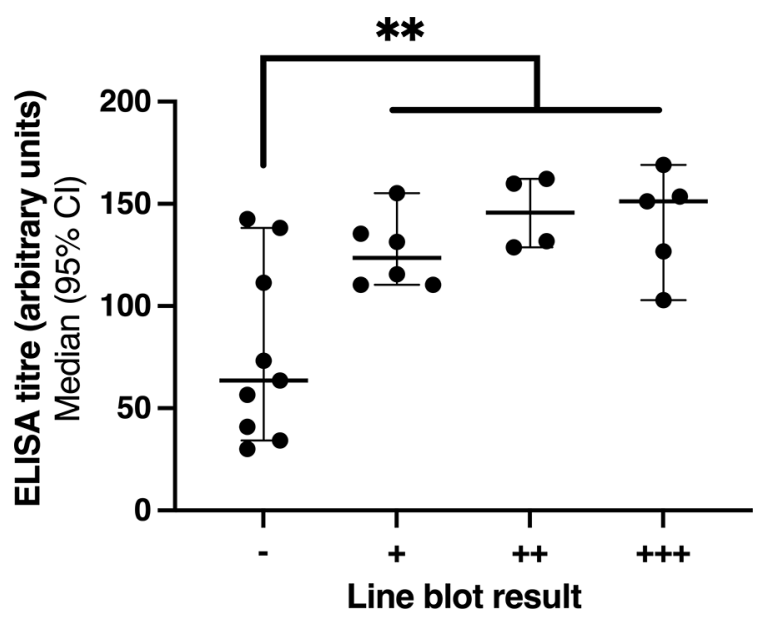

FIGURE 2 | Comparison of anti-TIF1 $\gamma$ ELISA titre and line blot result. ${ }^{\star \star} \mathrm{P} \leq 0.01$. 
positives out of the anti-TIF1 $\gamma$ samples that tested positive by ELISA and immunoprecipitation.

Graph showing a comparison between anti-TIF1 $\gamma$ ELISA titre and line blot result, as previously tested by our group (7). ELISA titres are expressed in arbitrary units and calculated as per the manufacturer's instructions. Lines and error bars represent median values with 95\% confidence intervals. The line blot results are expressed as negative $(-)$, low positive $(+)$, moderately positive $(+$ $+)$, and high positive $(+++)$. The median ELISA values for negative, low positive, moderately positive, and high positive results were 63.5 au., 123.5 au., 145.8 au., and 151.2 au., respectively. A two-tailed Mann Whitney test comparing ELISA titres between negative (-) and positive $(+,++,+++)$ line blots found a statistical difference between the two groups $(\mathrm{P}=0.0041)$.

\section{DISCUSSION}

This data has shown that accurate detection of anti-TIF1 $\gamma$ can be achieved by ELISA and confirms the findings made by Fujimoto et al. (10). The accuracy of detection is high and would be acceptable for use in clinical practice. Compared to other costand time- effective methods such as line and dot blot which have false negative rate of $40 \%-70 \%$ (7), this data found that ELISA has a false negative rate of $1 / 42(2 \%)$. This data has also shown that anti-TIF1 $\gamma$ titre correlates with a positive line blot result. This result is not unexpected given that the line blot is a semiquantitative method of detecting autoantibodies. Importantly, where ELISA was able to detect samples with low titres of antiTIF1 $\gamma$ (between 30 - 100 au.), line blot was unable to do so. Line blot also failed to detect some samples with high anti-TIF1g titres (> 100 au.). Taken together, anti-TIF1 $\gamma$ ELISA performs better than line blot in detecting this clinically important autoantibody.

Anti-TIF1 $\gamma$ status by immunoprecipitation was determined by recognition of $155 / 140 \mathrm{kDa}$ bands alongside an anti-TIF1 $\gamma$ standard control. It remains possible that the sample negative by ELISA has an unknown autoantibody with an identical band pattern although this would seem unlikely. Furthermore, the sample produced an ELISA result just below the positive threshold and may simply be a low-titre positive. The ELISA threshold could be adjusted to reduce the likelihood of this occurring, but this is likely to lead to some false positives. The most appropriate cut-off threshold may depend on the clinical context, for example, a low false positive rate may be tolerable in patients with confirmed dermatomyositis to inform the intensity of malignancy screening.

The current study was not designed to investigate the relationship between anti-TIF1 $\gamma$ titres and cancer detection

\section{REFERENCES}

1. McHugh NJ, Tansley SL. Autoantibodies in Myositis. Nat Rev Rheumatol (2018) 14:290-302. doi: 10.1038/nrrheum.2018.56

2. Betteridge Z, Tansley S, Shaddick G, Chinoy H, Cooper RG, New RP, et al. Frequency, Mutual Exclusivity and Clinical Associations of Myositis Autoantibodies in a Combined European Cohort of Idiopathic rates. It would, however, be useful to investigate how antiTIF1 $\gamma$ titre using ELISA correlates with malignancy. Recent work by Fiorentino et al. (13) found anti-TIF1 $\gamma$ titre positively correlated with cancer detection rate in DM, ranging from $8 \%$ detection for low titres to $36 \%$ detection for high titres. Furthermore, some of our healthy control samples had low anti-TIF1 $\gamma$ titres just below the positive cut-off and it would be of interest to investigate if these healthy subjects had a higher malignancy rate compared to a negative anti-TIF1 $\gamma$ control population.

The detection of anti-TIF1 $\gamma$ in adult DM patients should be considered a red flag for malignancy (4-6). Accurate and timely detection of anti-TIF1 $\gamma$ autoantibodies is therefore vital for these patients to ensure underlying malignancy is diagnosed and treated promptly. We suggest that, when investigating IIMs, anti-TIF1 $\gamma$ ELISA is undertaken alongside, ANA testing and a multiplex immunoblot assay to ensure accurate detection of this important autoantibody.

\section{DATA AVAILABILITY STATEMENT}

The raw data supporting the conclusions of this article will be made available by the authors, without undue reservation.

\section{ETHICS STATEMENT}

The studies involving human participants were reviewed and approved by University of Bath EIRA 17-01211. Written informed consent to participate in this study was provided by the participants' legal guardian/next of kin.

\section{AUTHOR CONTRIBUTIONS}

Experiments were carried out by BM, HL, FM, and DL. Manuscript was drafted by BM and read by ST and NM. All authors contributed to the article and approved the submitted version.

\section{FUNDING}

This work was supported by funding from CureJM and the Bath Institute of Rheumatic Diseases. ELISA kits were provided by MBL.
Inflammatory Myopathy Patients. J Autoimmun (2019) 101:48-55. doi: 10.1016/j.jaut.2019.04.001

3. Aussy A, Boyer O, Cordel N. Dermatomyositis and Immune-Mediated Necrotizing Myopathies: A Window on Autoimmunity and Cancer. Front Immunol (2017) 8:992. doi: 10.3389/fimmu.2017.00992

4. Oldroyd A, Sergeant JC, New P, McHugh NJ, Betteridge Z, Lamb JA, et al. The Temporal Relationship Between Cancer and Adult Onset Anti-Transcriptional 
Intermediary Factor 1 Antibody-Positive Dermatomyositis. Rheumatol (United Kingdom) (2019) 58:650-5. doi: 10.1093/rheumatology/key357

5. Hida A, Yamashita T, Hosono Y, Inoue M, Kaida K, Kadoya M, et al. AntiTIF1- $\gamma$ Antibody and Cancer-Associated Myositis: A Clinicohistopathologic Study. Neurology (2016) 87:299-308. doi: 10.1212/WNL.0000000000002863

6. Fiorentino DF, Chung LS, Christopher-Stine L, Zaba L, Li S, Mammen AL, et al. Most Patients With Cancer-Associated Dermatomyositis Have Antibodies to Nuclear Matrix Protein NXP-2 or Transcription Intermediary Factor $1 \gamma$. Arthritis Rheum (2013) 65:2954-62. doi: 10.1002/art.38093

7. Tansley SL, Li D, Betteridge ZE, McHugh NJ. The Reliability of Immunoassays to Detect Autoantibodies in Patients With Myositis is Dependent on Autoantibody Specificity. Rheumatol (United Kingdom) (2020) 59:2109-14. doi: 10.1093/rheumatology/keaa021

8. Espinosa-Ortega F, Holmqvist M, Alexanderson H, Storfors H, Mimori T, Lundberg IE, et al. Comparison of Autoantibody Specificities Tested by a Line Blot Assay and Immunoprecipitation-Based Algorithm in Patients With Idiopathic Inflammatory Myopathies. Ann Rheum Dis (2019) 78:858-60. doi: 10.1136/ANNRHEUMDIS-2018-214690

9. Targoff IN, Mamyrova G, Trieu EP, Perurena O, Koneru B, O’Hanlon TP, et al. A Novel Autoantibody to a $155-\mathrm{Kd}$ Protein Is Associated With Dermatomyositis. Arthritis Rheum (2006) 54:3682-9. doi: 10.1002/art.22164

10. Fujimoto M, Murakami A, Kurei S, Okiyama N, Kawakami A, Mishima M, et al. Enzyme-Linked Immunosorbent Assays for Detection of Anti-Transcriptional Intermediary Factor-1 Gamma and Anti-Mi-2 Autoantibodies in Dermatomyositis. J Dermatol Sci (2016) 84:272-81. doi: 10.1016/j.jdermsci.2016.09.013

11. Tansley SL, Simou S, Shaddick G, Betteridge ZE, Almeida B, Gunawardena H, et al. Autoantibodies in Juvenile-Onset Myositis: Their Diagnostic Value and
Associated Clinical Phenotype in a Large UK Cohort. J Autoimmun (2017) 84:55-64. doi: 10.1016/j.jaut.2017.06.007

12. Fujimoto M, Hamaguchi Y, Kaji K, Matsushita T, Ichimura Y, Kodera M, et al. Myositis-Specific Anti-155/140 Autoantibodies Target Transcription Intermediary Factor 1 Family Proteins. Arthritis Rheum (2012) 64:513-22. doi: 10.1002/art.33403

13. Fiorentino DF, Gutierrez-Alamillo L, Hines D, Yang Q, Casciola-Rosen L. Distinct Dermatomyositis Populations are Detected With Different Autoantibody Assay Platforms. Clin Exp Rheumatol (2019) 37:1048-51.

Conflict of Interest: The authors declare that the research was conducted in the absence of any commercial or financial relationships that could be construed as a potential conflict of interest.

Publisher's Note: All claims expressed in this article are solely those of the authors and do not necessarily represent those of their affiliated organizations, or those of the publisher, the editors and the reviewers. Any product that may be evaluated in this article, or claim that may be made by its manufacturer, is not guaranteed or endorsed by the publisher.

Copyright (C) 2022 Mulhearn, Li, McMorrow, Lu, McHugh and Tansley. This is an open-access article distributed under the terms of the Creative Commons Attribution License (CC BY). The use, distribution or reproduction in other forums is permitted, provided the original author(s) and the copyright owner(s) are credited and that the original publication in this journal is cited, in accordance with accepted academic practice. No use, distribution or reproduction is permitted which does not comply with these terms. 